\title{
Is it sufficient to repeat LINEAR accelerator stereotactic radiosurgery in choroidal melanoma?
}

\author{
Furdova $\mathrm{A}^{1}$, Horkovicova $\mathrm{K}^{1}$, Justusova $\mathrm{P}^{1}$, Sramka $\mathrm{M}^{2}$ \\ Department of Ophthalmology, Medical School, Comenius University in Bratislava and University \\ Hospital Ruzinov, Bratislava, Slovakia. afrf@mail.t-com.sk
}

\begin{abstract}
OBJECTIVES: One day session LINAC based stereotactic radiosurgery (SRS) at LINAC accelerator is a method of "conservative" attitude to treat the intraocular malignant uveal melanoma.

METHODS: We used model Clinac $600 \mathrm{C} / \mathrm{D}$ Varian (system Aria, planning system Corvus version 6.2 verification IMRT OmniPro) with $6 \mathrm{MeV} X$ by rigid immobilization of the eye to the Leibinger frame. The stereotactic treatment planning after fusion of CT and MRI was optimized according to the critical structures (lens, optic nerve, also lens and optic nerve at the contralateral side, chiasm). The first plan was compared and the best plan was applied for therapy at C LINAC accelerator. The planned therapeutic dose was 35.0 Gy by $99 \%$ of DVH (dose volume histogram).

RESULTS: In our clinical study in the group of 125 patients with posterior uveal melanoma treated with SRS, in 2 patients (1.6\%) was repeated SRS indicated. Patient age of the whole group ranged from 25 to 81 years with a median of 54 TD was $35.0 \mathrm{~Gy}$. In 2 patients after 5 year interval after stereotactic radiosurgery for uveal melanoma stage T1, the tumor volume increased to $50 \%$ of the primary tumor volume and repeated SRS was necessary.

CONCLUSION: To find out the changes in melanoma characteristics after SRS in long term interval after irradiation is necessary to follow up the patient by an ophthalmologist regularly. One step LINAC based stereotactic radiosurgery with a single dose $35.0 \mathrm{~Gy}$ is one of treatment options to treat T1 to T3 stage posterior uveal melanoma and to preserve the eye globe. In some cases it is possible to repeat the SRS after more than 5 year interval (Fig. 8, Ref. 23). Text in PDF www.elis.sk.

KEY WORD: linear accelerator, stereotactic radiosurgery, melanoma, choroidal tumor.
\end{abstract}

\section{Introduction}

Uveal melanoma is a relatively rare type of cancer, but the most common and most aggressive type of intraocular tumor in adults. The incidence of intraocular tumors varies from 0.2 to 1.0. According to the Slovak National Cancer Registry, the incidence in Slovakia is 0.2 to 0.6 / 100000 inhabitants. Choroidal malignant melanoma is the most frequent uveal melanoma - over $75 \%$ (8).

Age and volume (size) of the tumor have been shown to be prognostic indicators following therapy for posterior uveal melanoma. Today, the ability to diagnose primary uveal melanoma is better due to modern diagnostic tools e.g. ophthalmological examination, ultrasound, computed tomography and magnetic resonance. Due to improved diagnostic methods over the past three decades, radiotherapy (external beam, charged particle or brachytherapy)

${ }^{1}$ Department of Ophthalmology, Medical School, Comenius University in Bratislava and University Hospital Ruzinov, Bratislava, Slovakia, and ${ }^{2}$ Department of Stereotactic Radiosurgery, St. Elisabeth Cancer Inst. and St. Elisabeth University College of Health and Social Work, Bratislava, Bratislava, Slovakia

Address for correspondence: A. Furdova, MD, Mgr, PhD, MPH, MSc, Department of Ophthalmology, Medical School, Comenius University, Bratislava, Pazitkova 4, SK-821 03 Bratislava, Slovakia.

Phone: +421248234607 has become the preferred treatment for most patients with uveal melanoma. Different radiation modalities are used as therapy modalities of posterior uveal melanoma $(9,10)$.

In the last three decades, the management of patients with choroidal melanoma has changed from radical enucleation towards globe sparing techniques. Alternatives to the radical enucleation vary from observation in the very early stage of choroidal melanoma to transpupillary thermotherapy, block-excision, endoresection with pars plana vitrectomy, brachytherapy using a variety of radioisotopes, external beam radiotherapy, charged particles, Leksellgama knife and stereotactic radiosurgery $(2,3)$.

Stereotactic radiation therapy (linear accelerator therapy) and gamma-knife radiosurgery provide good local control, with survival rates comparable with other treatments. Stereotactic radiosurgery of extracerebral lesions like uveal melanoma has been invented in the last two decades and is an alternative treatment for small and middle posterior choroidal melanoma. In this study, we assess the treatment of posterior uveal melanoma by one-day session of linear accelerator /LINAC/ based stereotactic radiosurgery (SRS).

\section{Methods}

Clinical study of 125 patients with posterior uveal melanoma treated with SRS in period 2001-2013 and analysis of patients 
of repeated SRS was necessary. Patient age of the whole group ranged from 25 to 81 years with a median of $54 \mathrm{TD}$ was $35.0 \mathrm{~Gy}$. In 2 patients after 5 year interval after stereotactic radiosurgery for uveal melanoma stage T1, the tumor volume increased to 50 $\%$ of the primary tumor volume and repeated SRS was necessary.

We report the clinical course of the disease in 2 patients with posterior uveal melanoma (choroid) in stage T1/T2 who underwent repeated stereotactic radiosurgery at LINAC linear accelerator in period 2001-2013 in Slovakia.

Magnetic resonance findings were basic to indicate the patient for linear accelerator therapy.

Immobilization of the eye with intraocular tumor was performed surgically by sutures to the stereotactic frame. Sutures were placed under local anesthesia to 4 direct extraocular muscles through conjunctiva and through the lids. The stereotactic frame was fixed to the head and the sutures were tied to the stereotactic Leibinger frame. The patient underwent computed tomography and magnetic resonance examination with the immobilized eye to the frame. Stereotactic radiosurgery was performed by one-day session on linear accelerator Model LINAC C 600 C/D Varian with $6 \mathrm{MV}$ $\mathrm{X}$. The stereotactic treatment planning scheme was created after fusion of CT and MRI findings and the final plan was optimized according to the critical structures - lens, optic disc, optic nerve, lens and optic nerve at the contralateral side, chiasm. Tumor volume calculation was based on the ROI (region of interest) of the tumor and $3 \mathrm{D}$ reconstruction was done. The planned therapeutic dose into the tumor mass was 35.0 Gy by $99 \%$ of DVH (dose volume histogram).

In the afternoon, the patient underwent irradiation at linear accelerator. Sutures and frame were removed. Next day, the patient underwent the examination by an ophthalmologist - the slit lamp examination, ophthalmoscopy, intraocular pressure measuring and was released for home treatment with local therapy (eye drops antibiotics, corticosteroids, lubricant).

The doses to the critical structures were below 8.0 Gy for the optic nerve and the optic disc and 10.0 Gy to the anterior segment of the eye.

After stereotactic radiosurgery, patients were referred regularly in six month interval to their oncologist to screen metastasis (liver ultrasound, abdominal ultrasound, liver's function test; once per year chest X-ray), some patients were recommended to whole body PET/CT (positron emission tomography).

\section{Results}

The total number was 125 patients with posterior uveal melanoma treated with SRS in period 2001-2013, patient age ranged from 25 to 81 years, with a median of 54 TD was 35.0 Gy. In 2 patients $(1.6 \%)$ after 5 year interval after primary stereotactic radiosurgery for uveal melanoma stage $\mathrm{T} 1$, the tumor volume increased to $50 \%$ of the primary tumor volume and repeated SRS was indicated. In second SRS treatment, the same TD of 35.0 Gy was used.

Patients were examined by an ophthalmologist (slit lamp examination, intraocular pressure, ophthalmoscopy, fundus photo and also ultrasound B-scan and optical coherence tomography) every 3 months in first year, later in 6 month interval after stereotactic radiosurgery.

\section{Clinical findings in patient 1}

In 2005, female patient born in 1939 was sent to our Department with uveal melanoma stage T1with maximum elevation of the tumor $4.7 \mathrm{~mm}$ (Fig. 1).

Stereotactic radiosurgery was performed in 2006 with 35.0 Gy TD.

Patient was observed regularly and in 2009 there was no sign of increased volume of the tumor.

Visual acuity (BCVA - best corrected visual acuity) was 6/12 Snellen chart. In 2 years interval after SRS, the patient underwent phakoemulsification of cataract with an artificial intraocular lens. Visual acuity was again $6 / 12$ Snellen chart. The volume of the tumor was reduced, maximum elevation was $3.5 \mathrm{~mm}$.

After 7 years interval after the primary irradiation by SRS with TD of 35.0Gy in 2013, the tumor maximum elevation was $7.7 \mathrm{~mm}$ and the volume increased over $50 \%$. Repeated SRS was indicated (Fig. 2).

Patient was admitted for repeated one day session stereotactic radiosurgery by linear accelerator with single dose TSD 35.0Gy.

After repeated SRS, the tumor elevation was still $4.0 \mathrm{~mm}$ in 2015. There were no signs of distant metastatic disease. Due to post-irradiation complications in macula and optic neuropathy visual acuity reduced to $6 / 60(0.1)$ Snellen chart.

\section{Clinical findings in patient 2}

In 1998 a male patient born in 1951 was sent to our Department with small uveal melanoma stage T1. He underwent brachytherapy with $\mathrm{Ru}^{106}$ plaque (Fig. 3).

After 3 years, the tumor volume increased and stereotactic radiosurgery was indicated - SRS with TD of 35.0Gy.

In 2001, due to an increased volume of the tumor, he was admitted to one day session stereotactic radiosurgery irradiation with uveal melanoma stage T1. Visual acuity (BCVA - best corrected visual acuity) was $6 / 18$ Snellen chart.

After stereotactic irradiation, the volume of the tumor was reduced, maximum elevation was $2.5 \mathrm{~mm}$. Secondary cataract developed and secondary glaucoma was treated by eye drops. Patient rejected cataract surgery (Figs 4 and 5).

In 2013 , the tumor elevation was $7.5 \mathrm{~mm}$ and the volume increased $50 \%$. Repeated SRS with TD 35.0 Gy at linear accelerator was indicated (Fig. 6).

After repeated SRS, the tumor elevation is still $3.5 \mathrm{~mm}$ in 2015. Due to post-irradiation complications and secondary neovascularisation, visual acuity reduced to "no light perception" (Fig. 7).

In 2016 patient, underwent enucleation of the blind eye due to secondary glaucoma.

The pathologist's macroscopic findings: crossed eyeball, tumor is not macroscopically visible, the sample has been processed completely (Fig. 8). 


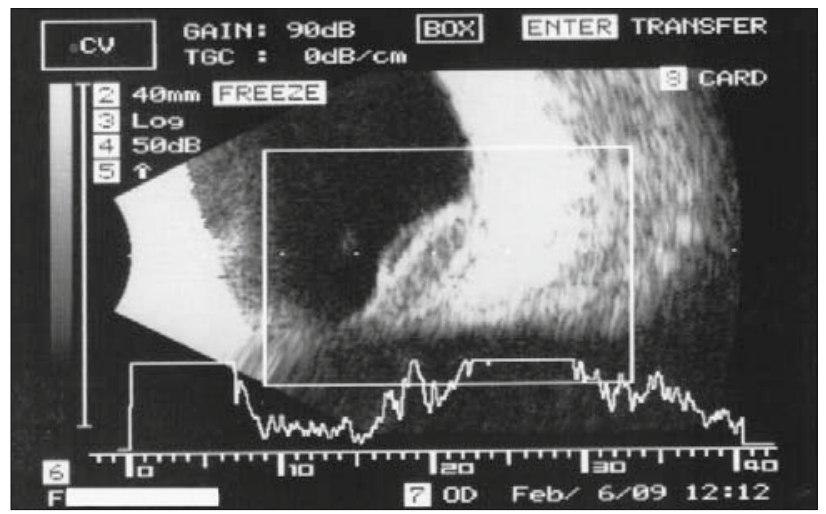

Fig. 1. Ultrasound finding of the tumor in 2009.

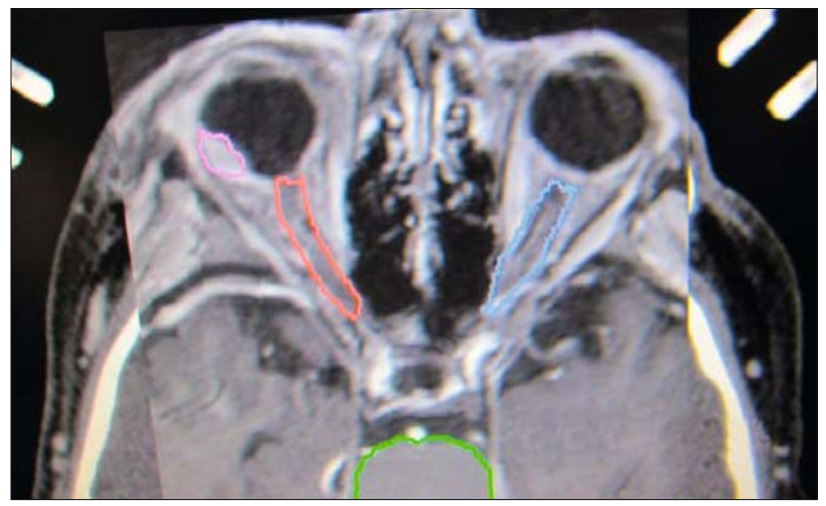

Fig. 2. Magnetic Resonance finding of intraocular uveal melanoma (pink color) - relapsed choroidal melanoma in 2013 before repeated SRS.

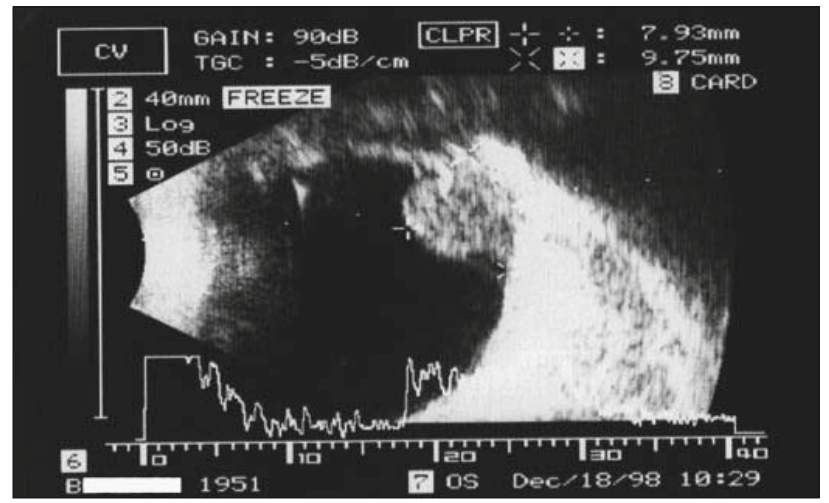

Fig. 3. Ultrasound finding of the tumor in 1998 with elevation $7.9 \mathrm{~mm}$.

The pathologist's microscopic findings: the eyeball processed completely. The cornea is with peeling of epithelium. On the edge is a chronic inflammatory reaction with proliferation of more vessels. In several places there are the regressive changes of the choroid and the wall of the eyeball in terms of fibrosis or granuloma resorption. Iris is slightly fibrotic, smoothed, outlining the adhesions of the anterior chamber angle, trabecular channels are identified.

Microscopically was captured one point with malignant melanoma G2, spindle cell type, with acinus ( 4 × 4 × $2 \mathrm{~mm}$ ), infiltrating

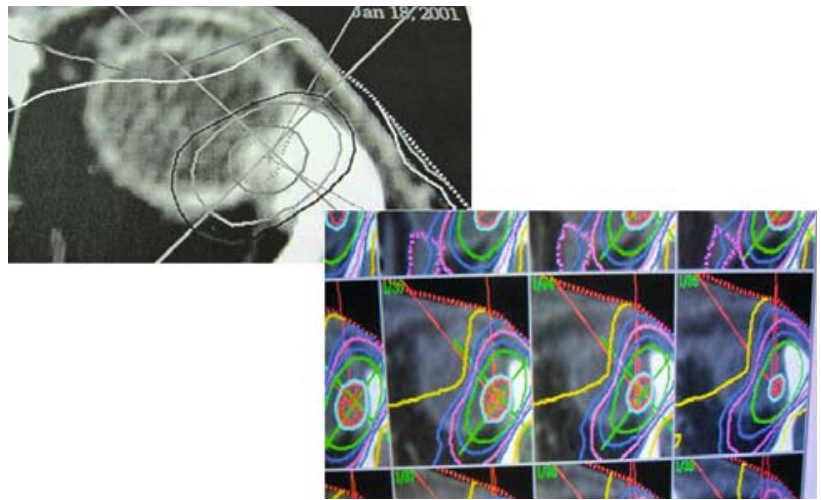

Fig. 4. Stereotactic planning scheme of the tumor in 2001.

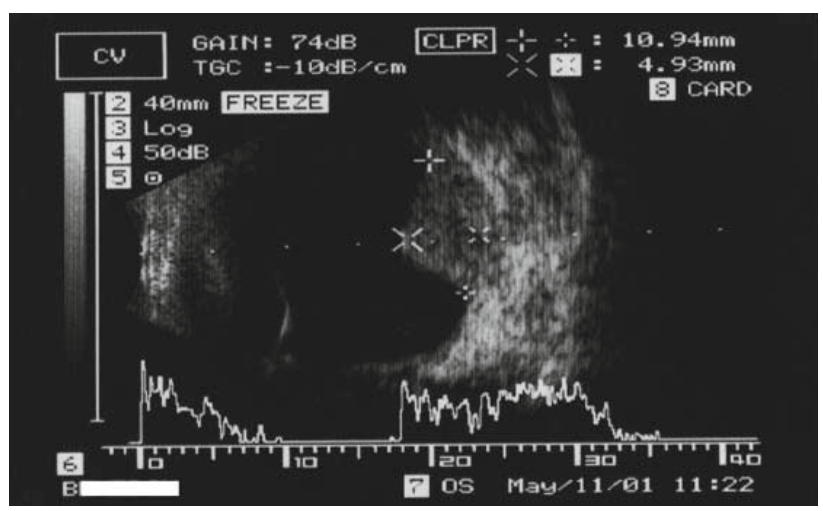

Fig. 5. Ultrasound finding after stereotactic irradiation in 2001.

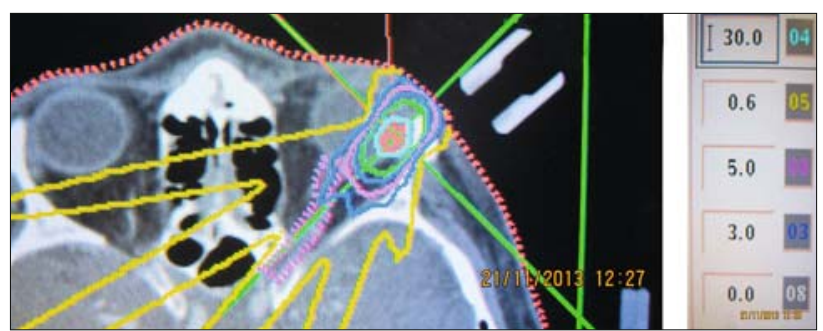

Fig. 6. Planning Scheme for repeated SRS in 2013 - tumor localized in choroid (red color).

uvea and inner edge of the eyeball wall. The blood-vessel invasion has not been observed. Immunophenotype: $\mathrm{S} 100+$, melanoma + , + HMB45, Bcl2 +, CD117 +, p53, cyklinD1-, Ki67 + $1 \%$.

Summary: One microscopic malignant melanoma captured, spindle cell G2 type B (with acinus), the infiltration of the inner wall surface of the eyeball; vascular infiltration or promotion to n.opticus has not been observed. Immunophenotype is with a favorable prognosis.

There are no signs up to date of metastatic disease in the patient. The patient was recommended to whole body PET/CT (positron emission tomography computed tomography) with negative results. 


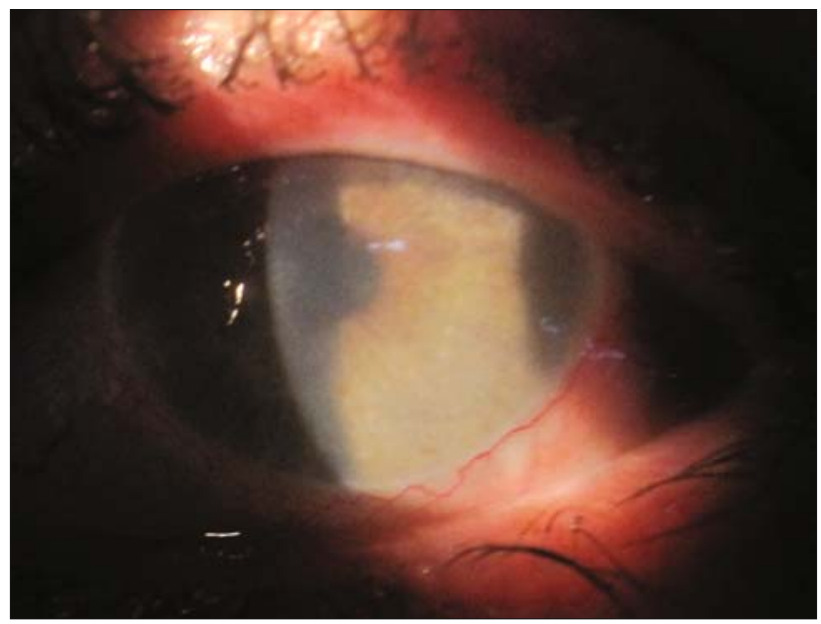

Fig. 7. Macro photo of the anterior segment of the eye with secondary glaucoma in 2014.

\section{Discussion}

For over 15 years, stereotactic photon beam irradiation has been under clinical investigation for the treatment of uveal melanoma. The therapeutic single dose has been reduced to as low as 35.0 Gy over the past few years without reduction in tumor control $(9,10)$. This dose is used in our study. Doses of 40.0 Gy delivered at the $50 \%$ isodose result in good local tumor control and acceptable toxicity. Since radiobiological studies indicate a possible advantage of hypofractionated treatment over a single very large fraction to sterilize uveal melanoma cell lines, fractionated stereotactic radiotherapy (SRT) has gained additional interest. Linear accelerators (LINAC) have the advantage of a feasible fractionation. A hypofractionated scheme of 4-5 fractions and total doses between 50.0 and 70.0 Gy employ the LINAC studies. In different studies for uveal melanoma has been proven that SRT reported over $90 \%$, 5 and 10 years after treatment, local tumor control rates $(6,7)$.
High rates of local control can be achieved with 5-year control rates exceeding $95 \%$ in patients treated with charged particles. Proton beam radiotherapy with a $62 \mathrm{MeV}$ cyclotron achieves high rates of local tumor control and eye preservation; visual acuity is depending on tumor stage - tumor size and location $(4,13)$.

Large, prospective, randomized trials were designed to compare mortality figures for medium-sized melanomas treated by brachytherapy or enucleation (1).

There has been performed no multicentre trial to assess dosimetry, safety and efficacy of SRS, or to evaluate outcomes of gamma knife radiosurgery for melanoma yet, but data from several reported case series suggest that SRS can have similar local tumor control rate, metastasis rate, mortality rate and complications rate when compared to brachytherapy. Recent studies have suggested that gamma knife radiosurgery and SRS may be an appropriate alternative for treating uveal melanoma in those patients, in whom lesions are ineligible for conventional brachytherapy. The findings in the series suggest a role of SRS in the treatment of selected cases of uveal melanoma.

Radiogenic side effects after stereotactic radiotherapy are cataract radiation retinopathy development, cataract, opticopathy and neovascular glaucoma. They result to secondary visual acuity losses and in some cases it is necessary to perform secondary enucleation. Overall, stereotactic photon beam radiotherapies (SRS and SRT) are considered effective treatment modalities for uveal melanoma, with promising late tumor control and toxicity rates. SRS is a relatively new method, so there is a need for multi-center trial to compare the outcomes following stereotactic radiosurgery with other methods. Stereotactic photon therapy of uveal melanoma, based on $\mathrm{CT}$ and MRI images, is a safe and precise treatment option. Local control was found to be excellent. LINAC based stereotactic irradiation for uveal melanoma is feasible and well tolerated and can be offered to patients with medium sized and unfavorably located uveal melanoma who are searching for an eye-preserving treatment. Because of selection criteria, the number of patients in the study with reduced visual acuity will probably increase in future (5).
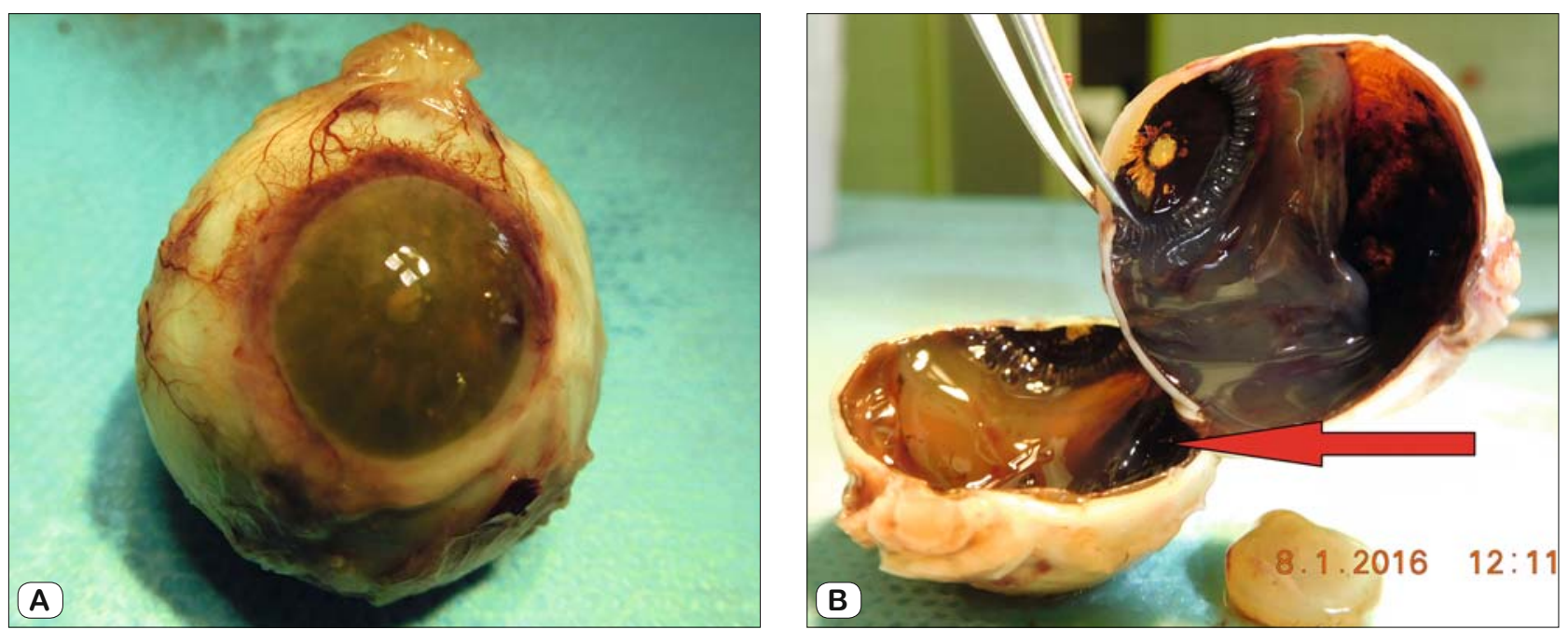

Fig. 8. Macro photo of the enucleated eye globe (A) in 1/2016 and dissected eye globe after enucleation (B), the arrow is pointing to the tumor mass. 
456-462

The retrospective study of Meyer et al. pointed out that irradiation of 30.0 Gy of more than $2 \mathrm{~mm}$ of the optic nerve head initiated an optic neuropathy (18).

Recent studies have suggested that gamma knife radiosurgery and SRS may be an appropriate alternative for treating uveal melanoma in those patients, in whom lesions are ineligible for conventional brachytherapy (20). The findings in the series suggest a role of SRS in the treatment of selected cases of uveal melanoma.

The eye retention is one of the main goals of the conservative treatment, but in some cases enucleation can be indicated due to complications after therapy e.g. secondary neovascular glaucoma $(12,15)$. In our study, we had the same experience, because in our case report the patient underwent enucleation due to secondary glaucoma (Figs 3, 4 and 5).

Lantigua-Dorville reported a case report of a 68 -year-old man diagnosed with choroidal melanoma in the right eye, who underwent treatment with episcleral brachytherapy (I125) and transpupillary thermotherapy. Ultrasound, computed tomography, and magnetic resonance imaging were performed and revealed ocular recurrence of choroidal melanoma. Treatment with extended enucleation was performed. Macroscopic and microscopic examinations revealed extraocular extension and malignant cells, respectively. Immunohistochemistry demonstrated tumor MelanA and HMB-45 expression. No cytogenic abnormalities were detected with fluorescence in situ hybridization of tumor cells using probes against chromosomes $3 \mathrm{q} 27$ and $8 \mathrm{q} 24$. The patient underwent adjuvant external beam radiotherapy for treatment of residual tumor tissue. This case represents the first reported case of recurrent choroidal melanoma with no cytogenetic abnormalities and the absence of metastatic disease, despite a number of the poorest prognostic factors (16).

Shiels et al in the retrospective, nonrandomized, interventional case series of 8100 patients with uveal melanoma evaluated the patients for melanoma-related metastasis based on the patient race. The patient race was Caucasian $(\mathrm{n}=7918,98 \%)$, Hispanic $(\mathrm{n}=105,1 \%)$, Asian $(\mathrm{n}=44,<1 \%)$, or African American $(\mathrm{n}=$ $33,<1 \%$ ). On the basis of the race (Caucasian, Hispanic, Asian, and African American), significant differences were noted in the mean age at presentation $(58,48,44$, and 52 years; $\mathrm{p}<0.001)$, distance of posterior tumor margin to foveola $(5,5,6$, and $4 \mathrm{~mm}$; $\mathrm{Pp}<0.001)$, distance of posterior tumor margin to optic disc (5, $5,6$, and $4 \mathrm{~mm} ; \mathrm{p}<0.001)$, tumor base $(11,12,12$, and $13 \mathrm{~mm} ; \mathrm{p}$ $<0.001)$, tumor thickness $(5.4,7.1,6.5$, and $7.5 \mathrm{~mm}$; $<<0.001)$, intraocular hemorrhage $(10,14,11$, and $24 \% ; \mathrm{p}=0.02)$, and rupture of Bruch's membrane $(20,27,39$, and $36 \% ; p=0.001)$. On the basis of multivariate analysis, the rate of metastasis increased with an increasing age $(p<0.001)$, ciliary body location $(p<$ $0.001)$, increasing tumor base $(\mathrm{p}<0.001)$, increasing tumor thickness $(\mathrm{p}<0.001)$, pigmented tumor $(\mathrm{p}=0.001)$, subretinal fluid $(p=0.001)$, intraocular hemorrhage $(p=0.045)$, and extraocular extension ( $\mathrm{p}=0.036)$. Kaplan-Meier estimates of metastasis at 3 , 5 , and 10 were 8,15 , and $25 \%$ in Caucasians; 13,13 , and $13 \%$ in Hispanics; 4, 4, and $36 \%$ in Asians; and 8, 8, and $8 \%$ in African Americans. Compared with Caucasians, despite relative risk for metastasis of 0.31 for African Americans, 0.73 for Hispanics, and 1.42 for Asians, there was no statistical difference in metastasis, or death from uveal melanoma based on race. In summary, uveal melanoma showed similar prognosis for all races (22).

The early detection of malignancy, particularly uveal melanoma, is crucial in protecting visual acuity, salvaging the eye, and preventing metastasis. Risk factors for early detection of uveal melanoma have been clearly delineated in literature and allow identification of melanoma when it is tiny and simulates a nevus. These factors include thickness $>2 \mathrm{~mm}$, presence of subretinal fluid (SRF), symptoms, the orange pigment, margin near optic disc, acoustic hollowness, surrounding halo, and absence of drusen. The importance of early detection is realized when one considers melanoma thickness, as each millimeter increase in melanoma thickness imparts $5 \%$ increased risk for metastatic disease. Newer imaging modalities like enhanced depth imaging optical coherence tomography and fundus autoflouroscence facilitate the detection of SRF and orange pigment. Additional molecular biomarkers and cytological features have been identified, which can predict the clinical behavior of a small melanocytic lesion. Features that suggest a poor prognosis include higher blood levels of tyrosinase m-RNA, vascular endothelial growth factor, insulin-like growth factor; monosomy 3 and gains in chromosome 8. Management of uveal melanoma includes enucleation (for large), local eye wall resection, brachytherapy, charged particle irradiation, and thermotherapy (for small to medium tumors). Although the role of a good clinical evaluation cannot be underestimated, it is advisable to evaluate the various radiological, molecular, and cytological features, to enhance the accuracy of early diagnosis and improved prognosis (21).

In the study of 7731 patients with posterior uveal (ciliary body and choroidal) melanoma, the AJCC (American Joint Committee on Cancer - 7th Edition ) tumor staging was stage I in 2767 (36\%), stage II in 3735 (48 \%), stage III in 1220 (16\%), and stage IV in $9(<1 \%)$. Based on tumor staging (I, II, III, and IV), features that showed a significant increase with tumor staging included the age at presentation $(57,58,60,60$ years $)(p<0.001)$, tumor base $(8,12,17,17 \mathrm{~mm})(\mathrm{p}<0.001)$, tumor thickness $(2.9,6.0,10.1$, $10.2 \mathrm{~mm})(\mathrm{p}<0.001)$, distance to optic disc $(3,5,5,5 \mathrm{~mm})(\mathrm{p}<$ $0.001)$, distance to foveola $(3,5,5,5 \mathrm{~mm})(\mathrm{p}<0.001)$, mushroom configuration $(6 \%, 24 \%, 34 \%, 33 \%)(\mathrm{p}<0.001)$, plateau configuration $(3 \%, 4 \%, 7 \%, 11 \%)(\mathrm{p}<0.001)$, tumor pigmentation $(48 \%, 53 \%, 69 \%, 78 \%)(\mathrm{p}<0.001)$, and extraocular extension $(0 \%, 1 \%, 11 \%, 22 \%)(\mathrm{p}<0.001)$. After therapy, Kaplan-Meier estimates of metastasis at $1,5,10$, and 20 years were $<1 \%, 5 \%$, $12 \%$, and $20 \%$ for stage I, $2 \% ; 17 \%, 29 \%$, and $44 \%$ for stage II; $6 \%, 44 \%, 61 \%$, and $73 \%$ for stage III, and $100 \%$ by 1 year for stage IV. Kaplan-Meier estimates of death at 1, 5, 10, and 20 years were $<1 \%, 3 \%, 6 \%$, and $8 \%$ for stage $\mathrm{I} ;<1 \%, 9 \%, 15 \%$, and $24 \%$ for stage II; $3 \%, 27 \%, 39 \%$, and $53 \%$ for stage III, and $100 \%$ by 1 year for stage IV. Compared to the stage I, the hazard ratio for metastasis/death was 3.1/3.1 for stage II and 9.3/10.1 for stage III. Conclusion of the study was that compared to uveal melanoma classified as AJCC stage I, the rate of metastasis/death was 3 times higher for stage II, 9 to 10 times 
higher for stage III, and even higher for stage IV. Early detection of posterior uveal melanoma, at a point when the tumor is small, can be lifesaving (23).

Optic neuropathy may follow radiosurgery in lesions near the visual pathways. Careful dose planning guided by MRI with restriction of the maximal dose to the visual pathways to less than 8.0 Gy will likely reduce the incidence of this complication (19).

Macular edema is the common complication after irradiation of uveal tumors. In the study of Mashayekhi et al, the aim was to determine the frequency of early subclinical macular edema in eyes with uveal melanoma and its association with future cystoid macular edema (CME). In the retrospective cohort study of totally 306 patients with uveal melanoma; 260 patients had follow-up of 1 or more years after plaque radiotherapy (follow-up cohort). The review of medical records and spectral-domain optical coherence tomography (OCT) images was published. Frequency of early subclinical macular edema (increased central macular thickness of $>10 \mu \mathrm{m}$ without cystoid changes before or at 4 months after plaque radiotherapy) was rated of future CME. In the results at baseline, 164 patients ( $54 \%$ ) had subclinical macular edema in the involved eye. On multivariate analysis, factors associated with subclinical macular edema at baseline were increasing tumor diameter $(p=0.001)$, increasing tumor thickness $(p=0.010)$, and subretinal fluid $(\mathrm{p}=0.001)$. Of 260 patients in the follow-up cohort, 105 (40\%) developed CME during a median follow-up of 31 months (mean, 34; range, 12-70 months). Eyes with subclinical macular edema at baseline (and at 4 months after plaque radiotherapy) had a significantly higher rate of future CME ( $\mathrm{n}=$ $66 ; 50 \%$ ) compared to eyes without subclinical macular edema at baseline $(\mathrm{n}=39 ; 30 \%)(\mathrm{p}=0.005$; hazard ratio, $1.77 ; 95 \%$ confidence interval, 1.19-2.64). In multivariate analysis, the factors associated with future development of CME included female gender $(p=0.004)$, increasing tumor thickness $(p<0.001)$, decreasing tumor distance to foveola $(p=0.002)$, hemorrhage over tumor $(p=0.017)$, and increased CMT of $>10 \%$ at baseline in the involved eyes compared to the opposite eyes $(p=0.012)$. Subclinical macular edema is common in eyes with uveal melanoma before and at 4 months after plaque radiotherapy and is associated with initial larger tumor size. Eyes with early subclinical macular edema are at significantly higher risk for future CME. These findings suggest that tumor-related factors, most likely mediated through proinflammatory cytokines, may play an important role in development of post-radiation CME (17).

In our study, in the mentioned 2 patients in the last 5 years we could not perform OCT examination due to cataract or secondary cataract in the pseudophakic eye and vitreous body opacities after stereotactic radiosurgery.

There are no signs up to date of metastatic disease in both our patients. They were recommended to whole body PET/CT (positron emission tomography computed tomography) with negative results.

Fractionated stereotactic radiotherapy and one day session stereotactic radiosurgery have emerged as promising, non-invasive treatments for uveal melanoma (14). Radiation therapy is now considered to be a useful component of the therapeutic armamentarium for malignant melanoma. Historically, melanoma has been considered a radioresistant tumor, but newer data have challenged this viewpoint. According to our results, a single one-day sessions SRS with 35.0 Gy is sufficient to treat small and middle stage melanoma (8).

Stereotactic radiosurgery with LINEAR accelerator is an alternative to enucleation in the treatment of uveal T1/T2 stage choroidal melanoma with a high tumor control. One day LINAC based stereotactic radiosurgery with a single dose of 35.0 Gy with a mechanical immobilization system with four sutures to extraocular muscles according to our study is a highly effective method to treat small and middle stage T1/T2 uveal melanoma $(9,10,11)$.

\section{Conclusions}

Repeated stereotactic radiosurgery for uveal melanoma in our group of patients was performed in 2 cases ( $1.6 \%$ ). Our first results in patients referred to repeated irradiation on linear accelerator showed a tumor regression by ultrasound and MRI examination. Follow - up of the patients is necessary by an ophthalmologist to find out the changes in intraocular melanoma characteristics after SRS in long term interval after irradiation.

One step LINAC based stereotactic radiosurgery with a single dose 35.0 Gy is one of treatment options to treat T1 to T3 stage posterior uveal melanoma and to preserve the eye globe and in some cases it is possible to repeat the SRS after more than 5 year interval. According to our results, one-day session SRS with 35.0 Gy is sufficient to treat small and middle stage choroidal melanoma.

\section{References}

1. Cohen VM, Carter MJ, Kemeny A, Radatz M, Rennie IG. Metastasis-free survival following treatment for uveal melanoma with either stereotactic radiosurgery or enucleation. Acta Ophthalmol Scand 2003; 81 (4): 383-388.

2. Collaborative Ocular Melanoma Study Group. Assessment of metastatic disease status at death in 435 patients with large choroidal melanoma in the Collaborative Ocular Melanoma Study. COMS Report No.15. Arch Ophthalmol 2001; 119 (5): 670-676.

3. Collaborative Ocular Melanoma Study Group. The COMS Randomized Trial of Iodine 125 Brachytherapy for Choroidal melanomas III. Initial Mortality Findings. COMS Report No. 18. Arch Ophthalmol 2001; 119 (7): 969-982.

4. Damato B, Kacperek A, Chopra M, Campbell IR, Errington RD. Proton beam radiotherapy of choroidal melanoma: the Liverpool-Clatterbridge experience. Int J Radiat Oncol Biol Phys 2005; 62 (5): 1405-1411.

5. Dieckmann K, Bogner J, Georg D, Zehetmayer M, Kren G, Pötter R. A linac-based stereotactic irradiation technique of uveal melanoma. Radiother Oncol 2001; 61 (1): 49-56.

6. Dieckmann K, Dietmar G, Zehetmayer M, Bogner J, Georgopoulos M, Potter R. LINAC based stereotactic radiotherapy of uveal melanoma: 4 years clinical experience. Radiother Oncol 2003; 67 (2): 199-206. 
456-462

7. Furdova A, Slezak P, Chorvath M, Waczulikova I, Sramka M, Kralik G. Radical surgical treatment (enucleation) or stereotactic radiosurgery in patients with posterior uveal melanoma - no difference in treatment outcome. Neoplasma 2010; 57 (4): 377-381.

8. Furdova A., Strmen P., Waczulikova I., Chorvath M., Sramka M., Slezak P. One-day session LINAC-based stereotactic radiosurgery of posterior uveal melanoma. Eur J Ophthalmol 2012; 22 (2): 226-235. doi: 10.5301/EJO.2011.77.

9. Furdova A, Ruzicka J, Sramka M, Kralik G, Chorvath M, Kusenda P. Choroidal melanoma stage T1 - comparison of the planning protocol for stereotactic radiosurgery and proton beam irradiation. Cesk Slov Oftalmol 2012; 68 (4): 156-161.

10. Furdova A, Sramka M, Chorvath M, Kralik G, Krasnik V, Krcova I et al. Stereotactic radiosurgery in intraocular malignant melanoma - retrospective study. Neuro Endocrinol Lett 2014; 35 (1): 28-36.

11. Furdova A, Sramka M. Uveal malignant melanoma and stereotactic radiosurgery: Intraocular uveal melanoma and one-day session stereotactic radiosurgery at linear accelerator. Saarbrücken: LAP LAMBERT Academic Publ., 2014, 181 p.

12. Ghazi NG, Ketcherside CS, Sheehan J, Conway BP. Gamma knife radiosurgery for uveal melanoma ineligible for brachytherapy by the Collaborative Ocular Melanoma Study criteria. Open Access Surg 2008; 1: 21-24.

13. Gragoudas ES, Lane AM, Munzenrider J, Egan KM, Li W. Longterm risk of local failure after proton therapy for choroidal/ciliary body melanoma. Trans Am Ophthalmol Soc 2002; 100: 43-49.

14. Henderson MA, Shirazi H, Lo SS, Mendonca MS, Fakiris AJ, Witt TC et al. Stereotactic radiosurgery and fractionated stereotactic radiotherapy in the treatment of uveal melanoma. Technol Cancer Res Treat 2006; 5 (4): 411-419.
15. Krema H, Somani S, Sahgal A, Xu W, Heydarian M, Payne D et al. Stereotactic radiotherapy for treatment of juxtapapillary choroidal melanoma: 3-year follow-up. Br J Ophthalmol 2009; 93 (9): 1172-1176. doi: 10.1136/bjo.2008.153429.

16. Lantigua-Dorville Y, Saornil MA, García-Alvarez C, GarcíaLagarto E. Choroidal melanoma recurrence after episcleral brachytherapy and transpupillary thermotherapy. Arq Bras Oftalmol 2016; 79 (1): 48-49.

17. Mashayekhi A, Schönbach E, Shields CL, Shields JA. Early subclinical macular edema in eyes with uveal melanoma: association with future cystoid macular edema. Ophthalmology 2015; 122 (5): 1023-1029.

18. Meyer A, Levy C, Blondel J, D’hermies F, Frau E, Schlienger P et al. Optic neuropathy after proton-beam therapy for malignant choroidal melanoma. J Fr Ophtalmol 2000; 23 (6): 543-553.

19. Mosci C, Mosci S, Barla A, Squarcia S, Chauvel P, Iborra N. Proton beam radiotherapy of uveal melanoma: Italian patients treated in Nice, France. Eur J Ophthalmol 2009; 19 (4): 654-660.

20. Mueller AJ, Talies S, Schaller UC, Horstmann G, Wowra B, Kampik A. Stereotactic radiosurgery of large uveal melanomas with the gamma knife. Ophthalmology 2000; 107 (7): 1381-1388.

21. Rishi P, Koundanya VV, Shields CL. Using risk factors for detection and prognostication of uveal melanoma. Indian J Ophthalmol 2015; 63 (2): 110-116.

22. Shields CL, Kaliki S, Cohen MN, Shields PW, Furuta M, Shields JA. Prognosis of uveal melanoma based on race in 8100 patients: The 2015 Doyne Lecture. Eye (Lond) 2015; 29 (8): 1027-1035.

23. Shields CL, Kaliki S, Furuta M, Fulco E, Alarcon C, Shields JA. American Joint Committee on Cancer Classification of Uveal Melanoma (Anatomic Stage) Predicts Prognosis in 7,731 Patients: The 2013 Zimmerman Lecture. Ophthalmology 2015; 122 (6): 1180-1186.

Received February 9, 2016. Accepted February 17, 2016. 